\title{
Suporte à dirigibilidade de um veículo elétrico através do gerenciamento de consumo de bateria
}

\author{
Gabriel dos Santos Nascimento ${ }^{1}$, José Maria N. David ${ }^{1}$, Mario A. R. Dantas ${ }^{1}$ \\ ${ }^{1}$ Departamento de Ciência da Computação -- Universidade Federal de Juiz de Fora \\ (UFJF)-- Juiz de Fora -- MG - Brazil \\ \{gabriel.nascimento, mario.dantas\}@ice.ufjf.br, jose.david@ufjf.edu.br
}

\begin{abstract}
This paper proposes an application to support the driver's awareness of the battery consumption of an electric vehicle and its driving modes. We simulated data capture using a context simulator capable of reproducing an electric vehicle's consumption and autonomy. We use Design Science Research (DSR) methodology to evaluate our proposed solution.
\end{abstract}

Resumo. Este artigo propõe uma aplicação para apoiar a percepção do motorista quanto ao consumo de bateria de um veículo elétrico, como também, aos modos de condução dele. Simulou-se a captação de dados através de um simulador de contexto capaz de reproduzir o consumo e autonomia de um veículo elétrico. Para avaliar nossa solução foi utilizada a metodologia de Design Science Research (DSR).

\section{Introdução}

Com o avanço de novas tecnologias, os veículos elétricos tendem a substituir os veículos à combustão. Contudo, a adoção de veículos elétricos enfrenta desafios como, por exemplo, a baixa autonomia, tempo de recarga e adaptação da rede elétrica para possibilitar o recarregamento residencial da bateria do veículo [Basit et al. 2020]. Além disso, outros fatores influenciam na durabilidade da carga da bateria como, por exemplo: congestionamento, sistema de controle climático e sistemas de entretenimento. Com isso, se faz necessário um entendimento a respeito dos mostradores de carga e da autonomia nos painéis de veículos elétricos [Lundström 2014].

A partir do exposto, percebe-se a necessidade da clareza e previsibilidade quanto ao nível de carga e autonomia ao conduzir um veículo elétrico. Para este fim, foi desenvolvida uma aplicação que capta dados de um veículo elétrico e fornece informações ao condutor como, por exemplo, histórico de consumo e de recarga, e nível de bateria. O objetivo deste trabalho é apresentar uma aplicação que capta os dados supracitados e os transforma em informações úteis para dar o suporte à decisão de recarregar o veículo, e se é possível realizar um novo trajeto com a carga atual de bateria. Além disso, este trabalho contribui para um dos grandes desafios de pesquisa em Sistemas de Informação no Brasil [Boscarioli et al. 2017], uma vez que aborda uma problemática relacionada diretamente ao desafio Conceito de Cidades Inteligentes no Brasil.

Este trabalho está organizado como segue. Na seção 2 apresentamos uma fundamentação teórica relativa ao desenvolvimento desta pesquisa. $\mathrm{Na}$ seção 3 
abordam-se a proposta e o desenvolvimento da solução. Na seção 4 discutimos os resultados da avaliação, e na seção 5 apresentamos a conclusão e trabalhos futuros.

\section{Fundamentação Teórica}

Com o objetivo de facilitar o entendimento do indicador de carga de um veículo elétrico, Lundström (2014) propôs o desenvolvimento de uma aplicação offline para dispositivo móvel capaz de fornecer informações mais precisas a respeito do indicador de carga da bateria veicular. $\mathrm{O}$ autor ressalta que este indicador sofre variação devido a fatores como o uso de sistema de climatização e congestionamento urbanos. O nosso trabalho propõe o desenvolvimento de uma aplicação para captar e enviar dados de consumo e nível de bateria em tempo real de um veículo elétrico em funcionamento.

Através de um estudo de níveis de estresse relacionado à condução de um veículo elétrico, Franke et al. (2012) conduziram uma pesquisa de campo que mapeou, por meio de um framework, a existência de um nível de autonomia confortável e variável pelo perfil do condutor. Os autores concluíram que se faz necessária uma readequação no conceito de uso e reabastecimento quando se conduz um veículo elétrico. O nosso trabalho, por sua vez, não dispõe de um framework, mas propõe uma aplicação que possui uma interface que visa expor os dados de consumo e nível de bateria do veículo de uma maneira lúdica e de fácil compreensão.

Por meio dos resultados dos autores supracitados, nosso trabalho propõe uma aplicação capaz de apoiar a familiarização do condutor com as peculiaridades e características de condução de um veículo elétrico como, por exemplo, suporte ao momento de decisão de recarregar o veículo.

\section{Metodologia de Pesquisa}

Como metodologia, utilizou-se Design Science Research (DSR) [Hevner et al. 2004]. Segundo Pimentel et al. (2018), o desenvolvimento de um artefato pode ser considerado como uma fonte de produção de conhecimento científico. Neste caso, o artefato é caracterizado pela aplicação idealizada, que visa dar suporte a um problema em um contexto real.

Portanto, através da DSR, foi possível identificar o problema por meio de um ciclo de design e idealizar uma solução. No ciclo de conhecimento ocorreu a estruturação do projeto e a execução do artefato em um ambiente simulado. Neste ciclo, utilizamos um software capaz de simular o funcionamento e as informações geradas através do deslocamento de um veículo elétrico. A escolha desse software foi pautada na sua facilidade de integração com a nossa aplicação, e pelo fato de ser open source.

O software de simulação escolhido, denominado Siafu (2007), dispõe de agentes customizáveis que interagem entre si e com o ambiente simulado. Este software tem sido utilizado com frequência em trabalhos do nosso grupo de pesquisa, como por exemplo, em [Do Nascimento et al., 2020]. A Figura 1 ilustra à esquerda um mapa que exibe o percurso do veículo. Além disso, à direita, no canto superior, podemos observar a demarcação de data e hora da simulação. No canto inferior da Figura 1, tem-se as informações específicas do veículo simulado como, por exemplo, posição geográfica, placa, consumo instantâneo e nível atual de bateria. Ademais, na Figura 1 ressalta-se 
que o consumo instantâneo do veículo é variável de acordo com o seu trajeto e a sua velocidade.

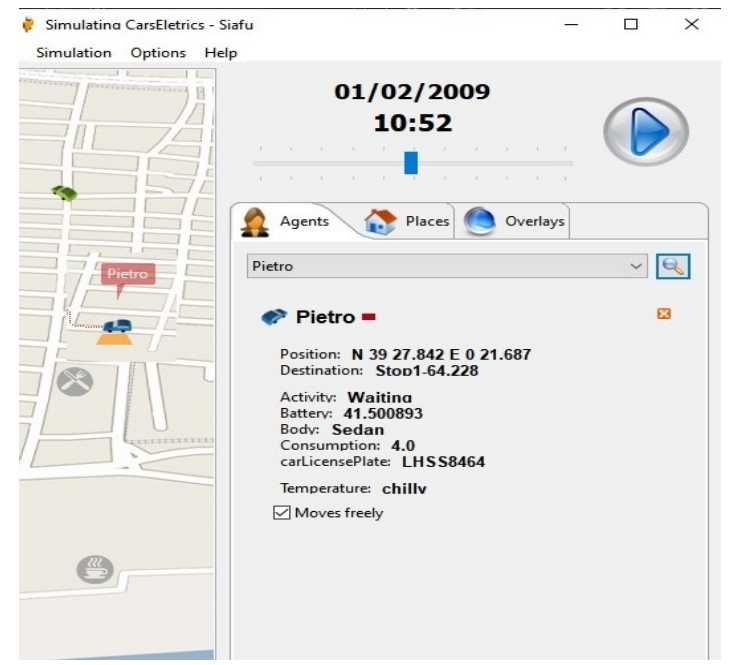

Figura 1 - Interface do simulador Siafu (2007)

\section{Resultados}

Após a implementação da solução e da utilização do simulador, uma avaliação inicial foi conduzida com o objetivo de capturar dados do veículo.
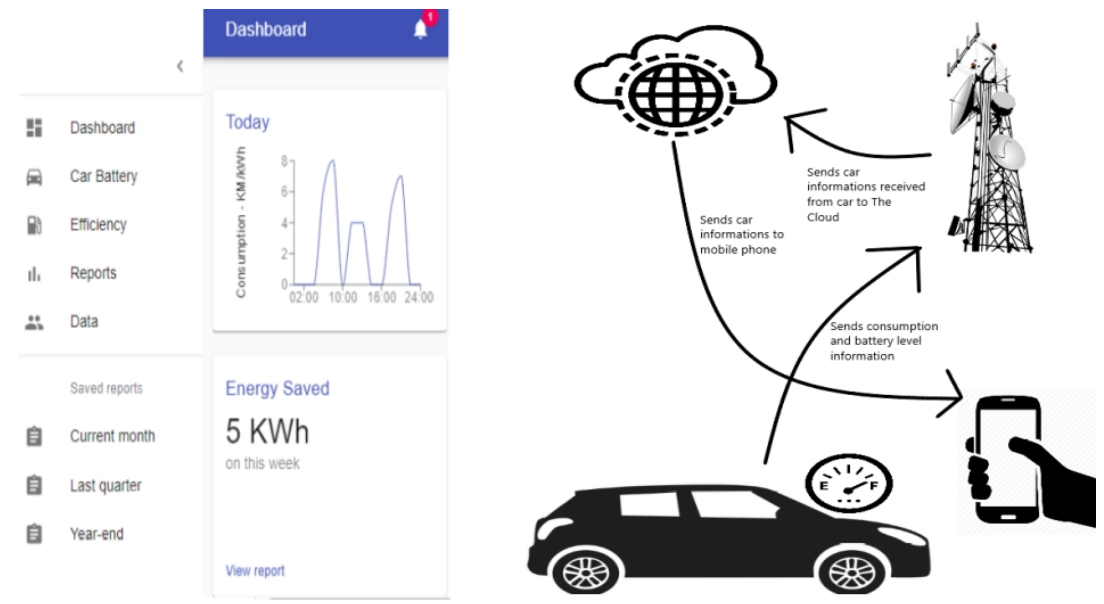

Figura 2 - Interface da aplicação e esboço de alto nível do projeto

Contudo, a execução do projeto em um contexto real acontece da seguinte forma: o veículo elétrico dotado de uma internet móvel envia os dados para um servidor na nuvem e, posteriormente, ele se comunica e armazena os dados em um banco de dados, também na nuvem. O usuário, por meio de uma aplicação instalada em um dispositivo móvel com acesso à Internet, consulta os dados fornecidos pelo seu veículo.

Na Figura 2, à esquerda, tem-se a aplicação desenvolvida neste projeto. Através dos gráficos, o usuário consegue visualizar a variação de consumo de seu veículo de acordo com as horas do dia. Além disso, a aplicação exibe informações do veículo e a quantidade de bateria economizada em uma semana. À direita, é possível observar o esboço do projeto, na prática, ou seja, um veículo elétrico envia os dados apresentados 
na Figura 1 para um servidor na nuvem e, posteriormente, a aplicação acessa o servidor e exibe os dados para o usuário.

\section{Conclusão}

Através da metodologia DSR, este trabalho de pesquisa apresentou uma aplicação para ampliar o suporte aos condutores de veículos elétricos. A aplicação é capaz de exibir informações em tempo real do veículo e auxiliar o condutor em relação ao momento de recarga, bem como se o veículo está apto a uma nova jornada. Nesse sentido, através da metodologia DSR, uma aplicação foi desenvolvida com o suporte de um simulador. Por meio das avaliações realizadas, pode-se tomar a proposta como base para futuros projetos na área de veículos elétricos.

Como trabalhos futuros, esperamos desenvolver uma interface para cada perfil de usuário. Além disso, cabe implementar funções de mapeamento de rota com o uso de algoritmos de caminho mínimo para minimizar o consumo de bateria durante o trajeto. Por fim, cabe avaliar a proposta de solução em um contexto real de condução do veículo.

\section{Agradecimentos}

O presente trabalho foi realizado com apoio do $\mathrm{CNPq}$ e da Coordenação de Aperfeiçoamento de Pessoal de Nível Superior - Brasil (CAPES) - Código de Financiamento 001.

\section{Referências}

Basit, A., et al. (2020) Electric vehicles interactions for efficient energy performance within smart grid. In: 2020 3rd International Conference on Computing, Mathematics and Engineering Technologies (iCoMET), pages 1-6, IEEE.

Boscarioli, C., Araujo, R., and Suzana, R. (2017). Grand research challenges in informa-tion systems in brazil 2016-2026.Brazilian Computer Society.

Do Nascimento, M. G., et al. (2020). Covid-19: A Digital Transformation Approach to a Public Primary Healthcare Environment. In 2020 IEEE Symposium on Computers and Communications (ISCC), pages 1-6, IEEE.

Franke, T., et al. (2012) Experiencing range in an electric vehicle: Understanding psychological barriers. Applied Psychology, 61(3):368-391.

Hevner, A. R., et al. (2004) Design science in information systems research. MIS quarterly, pages 75-105.

Lundström, A. (2014). Differentiated driving range: Exploring a solution to the problems with the "guess-o-meter" in electric cars. Proceedings of the 6th international conference on automotive user interfaces and interactive vehicular applications, pages $1-8$.

Pimentel, M., Filippo, D., Santoro, F. M. (2018). Metodologia de Pesquisa Científica em Informática na Educação: Concepção de Pesquisa. Vol. 1. Chapter 5, pages 1-29.

Siafu (2007). An open source context simulator. Disponível em: https://siafusimulator.org/. Acessado em: janeiro de 2020. 